\title{
A Simplified Historical-information-based SOC Prediction Method for Supercapacitors
}

\author{
Houlian Wang, Member, IEEE, Gongbo Zhou, Senior Member, IEEE, Jing Xu, Zhiqiang Liu, \\ Xiaodong Yan, Julie A McCann
}

\begin{abstract}
Range anxiety has become an important issue for the application of electric vehicles (EVs). Drivers need information on whether they can reach their destinations and what the remaining capacity would be before starting a trip. In order to satisfy the needs and save computing resources for computing-intense applications in vehicles, we propose a simplified historicalinformation-based State of Charge (SOC) prediction (SHSP) algorithm. First, definitions of SOC, historical average power, and equivalent current are given. Based on these definitions, Rintbased models of supercapacitors, under constant power and constant current loading, are established respectively. Then, a relationship between the historical average power and the predicted SOC is derived with the help of the equivalent current as a 'bridge'. The experimental results demonstrate that the 35step-forward SOC prediction error of the driving-behavior-based SOC prediction (SHSP) is close to the driving-behavior-based SOC prediction method (DBSP) and lower than Long-ShortTerm-Memory-based SOC prediction method (LSTM). Importantly, the time of running SHSP code is less than that of running DBSP code, and much less than that of running LSTM code.
\end{abstract}

Index Terms- SOC prediction; multi-step forward; supercapacitor; electrical vehicle; historical information

\section{INTRODUCTION}

$\mathrm{S}$ UPERCAPACITORS have high power densities, high efficiency, long lifespans, and respond quickly. Due to these advantageous characteristics, supercapacitors have become a popular energy storage device in electric vehicles, such as supercapacitor buses, subway, tram, and light rail vehicles.

For example, supercapacitor buses have been designed and used in Shanghai since 2006 [1]. These transit buses are solely powered by supercapacitors since the distance between two bus stations is no more than $20 \mathrm{~km}$ and supercapacitors can be

This work was supported in part by National Natural Science Foundation of China (No. 62002141), Natural Science Foundation for Colleges and Universities of Jiangsu Province (No.20KJB510052), Jiangsu Planned Projects for Postdoctoral Research Funds (No.2021K324C), National Natural Science Foundation of China (No.51905229), Natural Science Foundation of Jiangsu Province (No. BK20190968), China Postdoctoral Science Foundation (No.2019M661975) and Natural Science Foundation for Colleges and Universities of Jiangsu Province (No.19KJB460015). (Corresponding author: Gongbo Zhou ).

H. Wang is with School of Mechanical and Electrical Engineering, Jiangsu University of Science and Technology, Zhenjiang 221116, China, and also with Jiangsu Key Laboratory of Mine Mechanical and Electrical Equipment, China University of Mining \& Technology, Xuzhou 221116, China (whl@just.edu.cn) recharged quickly [2]. A supercapacitor-based light rail vehicle has also been developed, featuring improved efficiency and catenary-less operation [3]. A sightseeing car powered by supercapacitors is also designed for tourists [4].

Although supercapacitors have a lot of benefits, low energy density still leads to a smaller driving range compared with conventional vehicles. Limited driving range causes "range anxiety", which becomes a concerning topic for electric vehicle (EV) users [5]. For the aforementioned traffic tools, people repeat a relatively fixed journey from sites A and B, such as commuting. However, the initial state of energy storage elements in vehicles differs before each journey. It will cause a bigger judgment error about whether the vehicle can approach the destination only according to experience. Therefore, it is important to solve this problem.

State of Charge (SOC) is an important indicator to express the remaining capacity for supercapacitors, and SOC prediction is a potential method to solve "range anxiety". This is because drivers can know the future SOC before starting a new trip with the help of the SOC predictor. Then, drivers can decide to charge the vehicles or keep moving.

The current work of SOC prediction can be divided into two categories, one-step forward SOC prediction and multi-step forward SOC prediction. One-step forward SOC prediction can only predict the SOC the next time, and algorithms have to be executed recursively. Scholars presented many methods to predict one-step forward SOC, for example, Kalman filtering [6-8], Particle filter [9], and Luenberger-style algorithm [10]. However, the range of a single prediction step is limited, which plays a limited role in solving the range anxiety.

Multi-step forward SOC prediction is more important since SOC in future moments can be known before a journey. However, researchers pay less attention to this topic. There are two challenges. First, SOC is calculated based on current or

G. Zhou, X. Yan are with the Jiangsu Key Laboratory of Mine Mechanical and Electrical Equipment and School of Mechanical and Electrical Engineering, China University of Mining and Technology, Xuzhou 221116, China (gbzhou@cumt.edu.cn, yanxiaodong@cumt.edu.cn)

$\mathrm{J} . \mathrm{Xu}$ is with Marine Equipment and Technology Institute, Jiangsu University of Science and Technology, Zhenjiang 221116, China (xujing@just.edu.cn)

Z. Liu is with School of Mechanical and Electrical Engineering, Jiangsu University of Science and Technology, Zhenjiang 221116, China (zhiqiangliu@just.edu.cn)

J. McCann is with School of Computing, Imperial College London, London SW7 2AZ, UK (j.mccann@imperial.ac.uk). 
voltage. The future current and voltage are hard to be forecasted. Then the future SOC, of course, is not accessible. For example, Homan et al. [11] adopted DiBu-model to predict the battery voltage during charging, discharging, and idle periods. Then SOC is obtained according to the relationship between SOC and voltage. However, the future working conditions are hard to be obtained before using this model.

The other challenge is the current multi-forward SOC prediction method is relatively complex and time-consuming. This contradiction is that the capability of the onboard computer is limited for increasing applications. Not only SOC prediction but also new technologies, such as automatic driving [12], big data $[13,14]$, need computing resources. Also, waiting for the SOC prediction process will raise anxiety and irritability, which may have a negative on driving safety [15]. Currently, Dineva et al. [16] sampled discharge profiles obtained at different Crates, and then applied machine learning to forecast multi-step SOC. Hong et al. [17] used Long Short-Term Memory (LSTM) to perform multi-forward-step SOC prediction by taking into account weather and drivers' driving behaviors. However, machine learning-based methods are usually time-consuming. We also proposed a driving-behavior-based SOC prediction method (DBSP) based on a Rint model with a liner capacitor [18].

In response to the above two limitations, we use historical information to predict the driver's behavior in a future trip. We transform fluctuant variables into constants in a discharge process to simplify the calculation based on a simpler model. In detail, we use historical average power as the average power in the future trip. We do not predict future current, but try to transform the future current into a constant parameter, equivalent current, in a discharge process. Then, we connect average power and equivalent current, and also connect the equivalent current and future SOC. Finally, SOC can be predicted with historical average power. Then a simplified historical-information-based SOC prediction method (SHSP) is proposed. Compared with DBSP, a simplified model (Rint) with a constant capacitor is applied in this paper $[19,20]$ to reduce the computational complexity.

The contributions of this paper are as follows.

1. A Rint model with a constant capacitor for a supercapacitor is used. Based on this model, a multi-step forward SOC prediction method is presented in this paper. This method saves computational time, compared with DBSP and LSTM [17].

2. The linear approximation method is used to calculate the discharge current of supercapacitors at constant power loading. The calculation of energy consumed by the internal resistance becomes an integral process. The calculation is no longer an incremental iteration. This saves the computational time of equivalent current calculation within an endurable prediction error.

The remainder of this paper is divided into the following sections. In section II, definitions are introduced and explained. In section III, the SOC prediction framework is given. Section IV presents two equivalent circuits of a supercapacitor are built under two different working conditions. Section V presents the
SHSP method. In Section VI, the performance of SHSP is analyzed. In Section VII, the prediction performance is validated in experiments. Section VIII presents the conclusions of this paper.

\section{DeFINITIONS}

\section{A. State of Charge}

SOC represents the remaining charge stored in supercapacitors divided by its capacity. In order to simplify the derivation, capacity fading is neglected and the capacity is assumed to be constant. The SOC is usually calculated by using the ampere-hour method. Let $S O C_{0}$ represent the initial SOC. $Q_{0}$ is the capacity of the supercapacitor and $i_{n}$ is the practical discharge current for duration $t_{n}$ with coulombic efficiency $\eta_{n}$ for the trip. Let $S O C_{n}$ represent the future SOC discharged by the discharge current $\left(i_{1}, i_{2}, \ldots, i_{n}\right)$ and the expression is as follows.

$$
S O C_{n}=S O C_{0}-\left(\sum_{j=1}^{n} i_{j} t_{j} \eta_{j}\right) / Q_{0}
$$

\section{B. Historical average information}

Drivers' behavior is usually stable, which is the basis of SOC prediction in this paper. We use the simple method, the arithmetic means of the two parameters, to predict the future parameters, i.e., average power and driving time, for the next trip. Alternatively, the following references $[21,22]$ can be used to improve the prediction accuracy of the two parameters if readers are interested in the stochastic phenomenon of drivers' behavior.

Assuming drivers repeat the same trip, for example, the trip between site $\mathrm{A}$ and site $\mathrm{B}$. The historical average power and historical average driving time in these journeys can be calculated. Let $P_{h n}$ and $T_{h n}$ be the average power and average driving time in the $n$th trip, where $n$ is the number of trips. Driving time $T_{h n}$ can be calculated according to distance and speed. Assuming $P_{h n}$ and $T_{h n}$ satisfy a Gaussian probability distribution function, the expressions of historical average power $P_{\text {ha }}$, historical driving time $T_{\text {ha }}$ are shown as follows with standard deviation $\sigma_{P}$ and $\sigma_{T}$ respectively.

$$
\begin{aligned}
& P_{h \mathrm{a}}=\left(P_{h 1}+P_{h 2}+\ldots+P_{h n}\right) / n \\
& T_{\text {ha }}=\left(T_{h 1}+T_{h 2}+\ldots+T_{h n}\right) / n
\end{aligned}
$$

\section{Equivalent Current}

If the future SOC is wanted, it is better to obtain the current and coulombic efficiency at every moment in the next journey based on the formula (1). However, it is scarcely possible to acquire the future current. Neither the coulombic efficiency, since it changes with the current. If there is a constant replacing the variable (the real current), the solution procedure will be easier. Therefore, a virtual concept, i.e., equivalent current, is proposed to overcome the problem. The expression of the equivalent current is as follows, 


$$
I_{e}=(1 / T) \times \sum_{j=1}^{n} i_{j} t_{j} \eta_{j}
$$

where $T$ is the total discharge time and $T=\sum_{j}^{n} t_{j}$.

\section{SOC PREDICTION FRAMEWORK}

Based on the above definitions, a SOC prediction framework is shown in Fig. 1. First, the average power and the equivalent current are connected. This is the toughest part of this paper. Historical average power is estimated as the average power in the next discharge process. The future average power is viewed as a constant in a trip. Based on this, a constant power loading model will be built. The equivalent current is also constant in a trip. A constant current discharge model will be established, too. With the principle of energy conservation, the relationship between the average power and equivalent current can be obtained.

Second, the equivalent current and the SOC will be connected. This will be simpler since SOC can be interlinked to the equivalent current with formulas (1) and (4).

Finally, the average power and SOC are connected with the "bridge" of the equivalent current. Then, future SOC can be predicted.

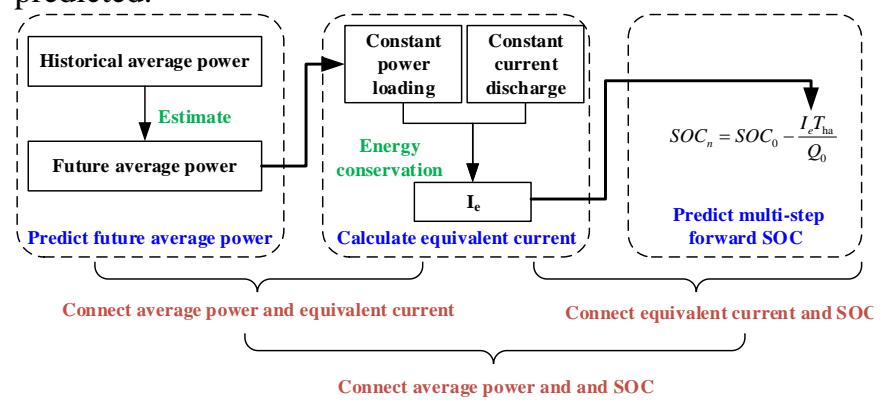

Fig. 1. The framework of SOC prediction method

\section{MOdELING}

Now, two models will be built in this section. The supercapacitor is modeled by using its simplest equivalent circuit because the difference in performance is not obvious between this model and other series-parallel branch models [23, 24], which can simplify the calculation.

\section{A. Constant Power Discharge Modeling}

Although real-time power in a trip varies, for the trip passed by, the average power in this trip can be seen as a constant in the overall discharge time because the average power is the arithmetic mean of the recorded power in this trip. A constant power loading is modeled for a future trip to simplify the prediction process (Fig. 2). The supercapacitor model is composed of capacitance $C$ and constant internal resistance $r_{\mathrm{p}}$. The ideal double-layer capacitance $C$ has an initial potential $U_{c o}$. The constant discharge power loading is represented and predicted by historical average power $P_{\text {ha }}$. The internal potential $U_{c}(t)$, terminal potential $U_{P}(t)$, and current during discharge $I_{P}(t)$ are all functions of the current time $t$.

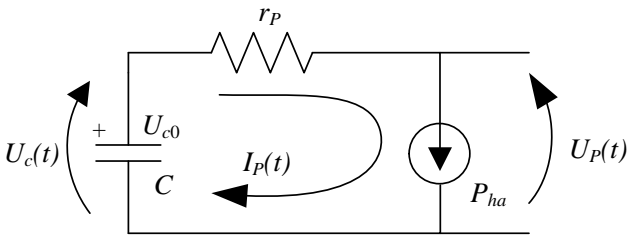

Fig. 2. Equivalent circuit of the supercapacitor under constant power loading

\section{B. Constant Current Discharge Modeling}

Fig. 3 shows the model of the supercapacitor under constant current discharge. The capacitance $C$, initial potential $U_{c o}$, and internal potential $U_{c}(t)$ are the same as those in Fig. 2. A constant discharge current loading is represented by the equivalent current $I_{e}$. The internal potential $U_{c}(\mathrm{t})$, terminal potential $U_{I}(t)$, and internal resistance $r_{I}(t)$ are functions of time. $r_{P}$ in the first model is the practical internal resistance and can be measured, while $r_{I}(t)$ in the second model is the virtual resistance and should be calculated.

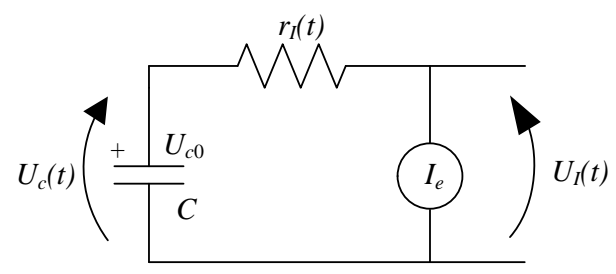

Fig. 3. The equivalent circuit of a supercapacitor under constant current discharge

\section{SOC PREDICTION APPROACH}

\section{A. Derivation of the SOC Prediction Approach}

To begin with, the relationship between the average power and the equivalent current must be established based on the above two models. The internal circuit in both models is the same, and the important difference between the two models is the load. To complement the transformation between two models, the energy consumed by two loads must be the same, and also the energy expended by two internal circuits must be identical. The expressions are as follows.

$$
\begin{aligned}
& W_{r_{P}}=W_{r_{I}} \\
& W_{P}=W_{I}
\end{aligned}
$$

where, $W_{r_{P}}$ and $W_{r_{I}}$ are energy consumed by the internal resistance $r_{\mathrm{P}}$ and $r_{I}(t)$ in the first model and the second model respectively. $W_{P}$ and $W_{I}$ are the energy consumed by the constant discharge power loading $P_{\text {ha }}$ and the constant discharge current loading $I_{e}$ respectively.

Formula (5) can then be calculated as formula (7) by integration,

$$
\int_{0}^{t} I_{P}(\tau)^{2} r_{P} d \tau=\int_{0}^{t} I_{e}{ }^{2} r_{I}(\tau) d \tau
$$

where $t$ is the time during discharge. The current $I_{P}(t)$ varies 
with time, and the internal resistance $r_{P}$ is unchanged under constant power loading in Fig. 2. By contrast, the current $I_{e}$ is a constant and the internal resistance $r_{I}(t)$ is variable under constant current discharge in Fig. 3.

Moreover, formula (6) can be expanded. In the first model, the output power is constant, and if the discharge time $t$ is known, the energy expended by constant power loading can be obtained as

$$
W_{P}=P_{\mathrm{ha}} t
$$

In the second model, the equivalent current is unchanged, but the terminal potential $U_{I}(t)$ varies. The energy expended by the constant current loading $W_{I}$ can be obtained by integrating $U_{I}(t)$ and $I_{e}$ over the interval $[0, t]$.

$$
W_{I}=\int_{0}^{t} U_{I}(\tau) I_{e} d \tau
$$

The terminal potential $U_{I}(t)$ can be given by subtracting $I_{e} r_{I}(t)$ from the internal potential $U_{c}(\mathrm{t})$ by Kirchhoff's Voltage Law (KVL).

$$
U_{I}(t)=U_{c}(t)-I_{e} r_{I}(t)
$$

The internal potential $U_{c}(\mathrm{t})$ is

$$
U_{c}(t)=U_{c 0}-\Delta U_{c}
$$

where $\Delta U_{c}$ is the difference between the initial potential $U_{c o}$ and the internal potential $U_{c}(t)$ at the time $t$, and the difference $\Delta U_{c}$ can be obtained as

$$
C \cdot \Delta U_{c}=I_{e} \bullet t
$$

Then make the substitution from (10)-(12) into (9) yields.

$$
W_{I}=U_{c 0} I_{e} t-\frac{I_{e}^{2} t^{2}}{2 C}-\int_{0}^{t} I_{e}{ }^{2} r_{I}(\tau) d \tau
$$

Formula (6) can be rewritten by substituting formulas (8) and (13),

$$
P_{\mathrm{ha}} t=U_{c 0} I_{e} t-\frac{I_{e}^{2} t^{2}}{2 C}-\int_{0}^{t} I_{e}^{2} r_{I}(\tau) d \tau .
$$

Finally, the equivalent current can be obtained by using formulas (7) and (14):

$$
I_{e}=\frac{U_{c 0} t-\sqrt{U_{c 0}^{2} t^{2}-2 \frac{t^{2}}{C}\left(P_{\mathrm{ha}} \times t+\int_{0}^{t} I_{p}(\tau)^{2} r_{p} d \tau\right)}}{\frac{t^{2}}{C}}
$$

when the following formula holds,

$$
U_{c 0}^{2} t^{2} \geq 2 \frac{t^{2}}{C}\left(P_{\mathrm{ha}} \times t+\int_{0}^{t} I_{p}(\tau)^{2} r_{p} d \tau\right) .
$$

The current $I_{p}(t)$ of the supercapacitor under constant power loading can be achieved from formulas (16) and (17), and the derivation is shown in the Appendix.

$$
I_{P}(t)=\frac{U_{c}(t)}{2 r_{P}}-\sqrt{\left(\frac{U_{c}(t)}{2 r_{P}}\right)^{2}-\frac{P_{\mathrm{ha}}}{r_{P}}}
$$

$$
\begin{aligned}
t= & \frac{C}{4 P}\left[U_{c 0}^{2}-U_{c}^{2}\right]+r_{P} C \ln \left\{\frac{U_{c 0} \sqrt{U_{c 0}^{2}-4 r_{P} P_{\mathrm{ha}}}}{U_{c} \sqrt{U_{c}^{2}-4 r_{P} P_{\mathrm{ha}}}}\right\} \\
& +\frac{C}{4 P}\left[U_{c 0} \sqrt{U_{c 0}^{2}-4 r_{P} P}-U_{c} \sqrt{U_{c}^{2}-4 r_{P} P_{\mathrm{ha}}}\right]
\end{aligned}
$$

We can solve formulas (16) and (17) by using the bisection method [25] and achieve current $I_{p}(t)$ at each time $t$. Then, the integral item $\int_{0}^{t} I_{p}(\tau)^{2} r_{p} d \tau$ can be calculated discretely[18]. However, if the prediction range is large, the computation time will increase.

Instead, we use the linear approximation method to solve this problem. The voltage of supercapacitors decreases while the current of supercapacitors increases under constant power loading. We approximatively assume the discharge current increases linearly. The initial discharge current under constant power loading is as follows,

$$
I_{p 0}=\frac{P}{U_{c 0}} .
$$

Let $I_{p 1}$ represent the discharge current at the next time, which is obtained by the bisection method. Then, the approximate discharge current can be calculated as follows,

$$
I_{p}(t)=\left(I_{p 1}-I_{p 0}\right) t+I_{p 0}
$$

Then, we have,

$$
\int_{0}^{t} I_{p}(\tau)^{2} r_{p} d \tau=\frac{1}{3}\left(I_{p 1}-I_{p 0}\right)^{2} r_{p} t^{3}+I_{p 0}\left(I_{p 1}-I_{p 0}\right) r_{p} t^{2}+I_{p 0}^{2} r_{p} t,
$$

and the equivalent current formula (15) is finally obtained.

Next, we need to use the 'bridge', equivalent current, to connect the power and SOC. Based on the definitions of the SOC and the equivalent current, SOC could be expressed as follows.

$$
S O C_{n}=S O C_{0}-\left(\sum_{j=1}^{n} i_{j} t_{j} \eta_{j}\right) / Q_{0}=S O C_{0}-\frac{I_{e} T_{\mathrm{ha}}}{Q_{0}}
$$

Finally, the historical average power is applied to represent the average power in the future trip to predict SOC.

\section{B. SOC Prediction Pseudocode}

The procedure for implementing the SOC prediction algorithm is as follows. Please note that the equivalent current is calculated only once before the trip.

TABLE 1

Pseudocode of SHSP

\section{SHSP Algorithm}

1: Initialize the historical average power $P_{\text {ha }}$, prediction time $T_{\text {ha }}$, initial state of charge $S O C_{0}$, internal potential $U_{c}(0)$, and internal resistance $r_{\mathrm{P}}$.

2: Calculate the discharge current $I_{p 0}$ at the first time under constant power loading according to formula (18).

3: Calculate the discharge current $I_{p 1}$ at the first time under constant power by using the bisection method. 
4: Calculate the equivalent current $I_{e}$ according to formulas (15) and (20)

5: $\quad$ Predict the SOC according to formula (21)

\section{PERFORMANCE ANALYSIS}

\section{A. Time Complexity Comparisons}

Since formula (17) in SHSP is a transcendental equation. It is difficult to obtain the analytical solution and we plan to use the fzero function in Matlab to obtain the numerical solution. The fzero function is a combination of bisection, secant, and inverse quadratic interpolation methods [25]. Hence, the time complexity of SHSP is $\mathrm{O}(\log v) . v$ is the search interval in the bisection algorithm, the maximum value of $v$ is from the cut-off voltage to the initial voltage.

In contrast, Hong et al. [17] used an LSTM-based model to predict multi-forward-step SOC. The time complexity is $\mathrm{O}\left(\sum_{k}^{D} n_{k} \bullet d^{2}\right) . n$ is equal to the sequence length, $d=2$ is the representation dimension in the $k$ th layer, and $D=3$ is the number of layers.

The time complexity of DBSP is also $\mathrm{O}(n \log v)$. $n$ equals the prediction time range $(T)$. Compared with SHSP, DBSP has the item $\mathrm{K} /(6 P)\left[U_{c}^{3}+\left(U_{c}^{2}+4 \mathrm{r}_{p} P_{\mathrm{ha}}\right)^{3 / 2}\right]$ in the transcendental equation in the formula (18) in [18].

\section{B. Theoretical Error Analysis}

Let the prediction error between the SHSP predictor and real SOC is $\alpha$. Real SOC is obtained by the ampere-hour method according to formula (1). The $\alpha$ could be positive and negative. Let the prediction error between SHSP and DBSP is $\beta$, and the prediction error between the DBSP predictor and real SOC of supercapacitors is $\gamma$, where $\gamma$ could be positive and negative. Then, there is $\alpha=\beta+\gamma$. Since $\gamma$ cannot be modeled exactly, the value of $\gamma$ is inaccessible by calculation. Then, we will calculate $\beta$.

Let $Q_{\mathrm{S}}$ represent the capacity of the supercapacitor in this paper, and let $U_{\text {cut }}$ represent the cut-off voltage. We have $Q_{\mathrm{S}}=C U_{\mathrm{c}}-C U_{\text {cut }}$. Assuming $Q_{\mathrm{D}}$ represent the capacity of the supercapacitor in [18], $Q_{\mathrm{D}}=\left(C_{\mathrm{i}} U_{\mathrm{c}}+1 / 2 K U_{\mathrm{c}}^{2}\right)-\left(C_{\mathrm{i}} U_{\text {cut }}+1 / 2 K U_{\text {cut }}^{2}\right)$, where $C_{i}$ is a constant capacitor and $K$ is a gain. The difference in capacity is as follows.

$$
\Delta Q=-\frac{1}{2} K U_{c}^{2}+\left(C-C_{\mathrm{i}}\right) U_{\mathrm{c}}+\frac{1}{2} K U_{c u t}^{2}-\left(C-C_{\mathrm{i}}\right) U_{\mathrm{cut}}
$$

If the cut-off voltage is less than $\left(C-C_{\mathrm{i}}\right) / K$, When $U_{\mathrm{c}}=\left(C-C_{\mathrm{i}}\right) / K$, we have the maximum capacity difference $\Delta Q_{\max }$. Otherwise, when, $\Delta Q_{\max }$ can also be obtained. $U_{\mathrm{mc}}$ is the minimum voltage that can be discharged to the cut-off voltage in a whole discharge process. The exact expression is as follows.

$$
\Delta Q_{\max }=\left\{\begin{array}{l}
\left(C-C_{\mathrm{i}}\right)^{2} /(2 K)+\frac{1}{2} K U_{c u t}^{2}-\left(C-C_{\mathrm{i}}\right) U_{\text {cut }}, \quad U_{\text {cut }} \leqslant\left(C-C_{\mathrm{i}}\right) / K \\
-\frac{1}{2} K U_{m c}^{2}+\left(C-C_{\mathrm{i}}\right) U_{\mathrm{mc}}+\frac{1}{2} K U_{c u t}^{2}-\left(C-C_{\mathrm{i}}\right) U_{\text {cut }}, U_{\text {cut }}>\left(C-C_{\mathrm{i}}\right) / K
\end{array}\right.
$$

$\beta$ can then be obtained.

$$
\beta=\frac{\Delta Q_{\max }}{Q_{0}}
$$

\section{EXPERIMENTS}

\section{A. Model Parameter Identification}

Model parameters are identified at 4 different temperatures in this section. A supercapacitor module, MCP0165C00048R0SHZ is chosen and tested according to [26], and the supercapacitor is produced by Supreme Power Solutions Co., Ltd, Beijing, China. The experimental equipment consists of a NEWARE battery test system (BTS-8000), a temperature chamber, and a PC, as shown in Fig. 4. During the capacity test, the cut-off voltage is $29 \mathrm{~V}$. The values of the parameters are shown in Table 2.

TABLE 2 MODEL PARAMETERS

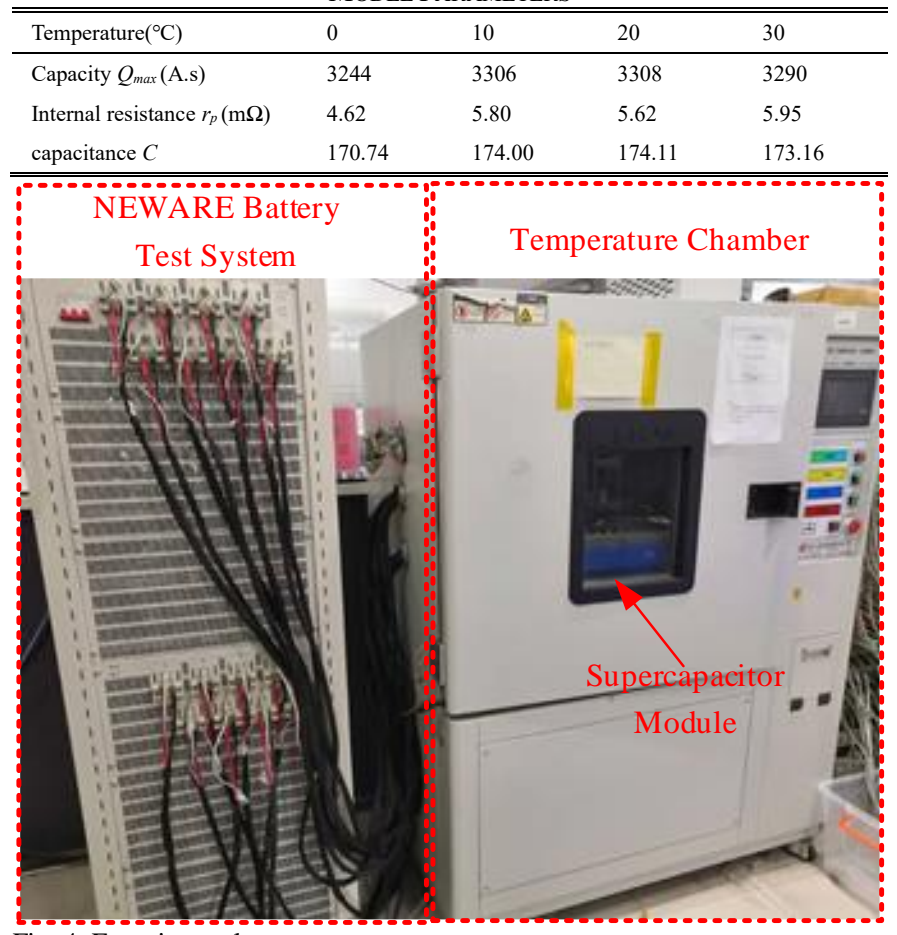

Fig. 4. Experimental apparatus

\section{B. Experimental Scheme}

All the working conditions are the same as the DBSP test conditions. The test routine consists of uphill sections, downhill sections, flat roads, and bends. For average power estimation at a new initial SOC, the interpolation method can be used based on historical information [18]. The sampling frequency of all the data is $10 \mathrm{~Hz}$. All the calculation is based on a laptop, with i7-77HQ, 8G RAM.

\section{Prediction Results}

First, the equivalent current is validated (Fig. 5). The experimental data in a discharge with $75 \%$ initial SOC is used. The prediction step is 2371 steps (237.1 seconds). Without consideration of the average power estimation error in this discharge, the estimated equivalent current is $10.28 \mathrm{~A}$ while the real equivalent current is $10.20 \mathrm{~A}$. The prediction error of the equivalent current is $0.78 \%$. The results prove the usefulness of 
the formula (15).

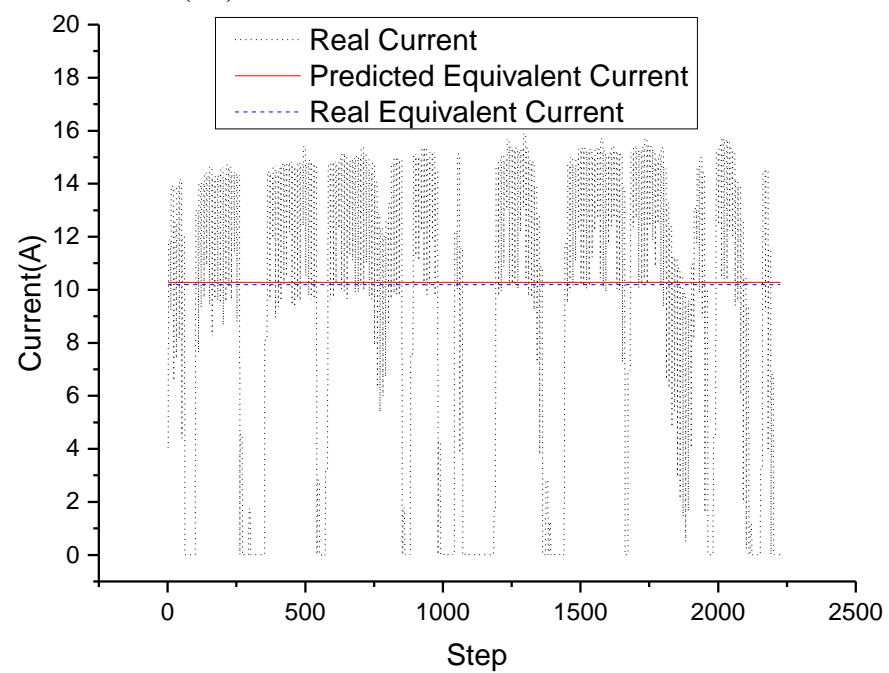

Fig. 5. Equivalent current at 2371 prediction steps with $75 \%$ initial SOC

Second, the SOC prediction accuracy and time consumption are also validated (Table 3 ) without the consideration of the average power estimation error. The running speed of SHSP code is fast. The SOC prediction accuracy is high with a relatively long prediction range. These results satisfy the application for EVs.

TABLE 3

SOC PREDICTION RESULTS AT 2371 PREDICTION STEPS

\begin{tabular}{lllll}
\hline \hline Initial SOC & $\begin{array}{l}\text { Real SOC at } \\
\text { the end }\end{array}$ & $\begin{array}{l}\text { Predicted SOC } \\
\text { at the end }\end{array}$ & $\begin{array}{l}\text { Prediction } \\
\text { error }\end{array}$ & $\begin{array}{l}\text { Time of } \\
\text { running SHSP } \\
\text { code (s) }\end{array}$ \\
\hline $75 \%$ & $1.84 \%$ & $1.25 \%$ & $0.58 \%$ & 0.011 \\
\hline \hline
\end{tabular}

D. Comparisons of SOC Prediction

To show the superiority of the presented method, SHSP, DBSP and LSTM are compared. Experimental data under $10^{\circ} \mathrm{C}$ are adopted here. SHSP and DBSP are executed based on the pseudocode in Tab. 1 and the pseudocode in [18] respectively. Setting parameters of LSTM are the same in [17], and we keep training window size/predicted window size $=60$ in this paper. The initial SOC is chosen as $32.9 \%$. We compared three methods in 7 limited forward steps from 5 to 35 .

We will take 35 steps forward prediction as an example to show how we compare the three methods. SHSP and DBSP will use historical information in past 4 trips (assuming we know) to calculate future power consumption. This is a simple way to show the prediction results. If the historical information is incomplete, the future power consumption can be obtained by interpolation. Based on the $32.9 \%$ initial SOC, the pseudocode of SHSP and DBSP is executed to predict 35 steps forward SOC. LSTM will use the past 2100 data $(35 \times 60)$ from time 1 to time 2100 to train the network. After the network is trained, 60 data from time 2101 to 2161 (the data at time 2161 is $32.9 \%$ ) are input into the network in order to output 35 steps forward prediction results.

TABLE 4

TIME OF RUNNING THE THREE METHODS AT 35 PREDICTION STEPS WITH $32.9 \%$ INITIAL SOC

\begin{tabular}{llll}
\hline \hline Methods & SHSP & DBSP & LSTM \\
\hline Time of running methods (s) & 0.011 & 0.191 & 0.860 \\
\hline \hline
\end{tabular}

Next, we start to compare the complexity of the three methods. We use the time of running the code of three methods (SHSP, DBSP, and LSTM [17]) to measure the complexity.

The time of running the three methods is shown in Tab. 4. It takes the shortest time for SHSP to predict the SOC and it takes the longest time for the LSTM-based method. The computational time for the DBSP code is about 17 times that of SHSP. This is because SHSP has fewer items and needs fewer iterations, saving more time. The computational time for the LSTM code is 78 times more than that of SHSP. This is because LSTM has a complex network structure.

Then, the prediction accuracy is compared. The ampere-hour method is used to obtain the real SOC, as shown in formula (1). Fig. 6 shows SHSP and DBSP are close to the real SOC. LSTMbased SOC prediction is fluctuant around the real SOC. Three methods achieve lower values than the real SOC.

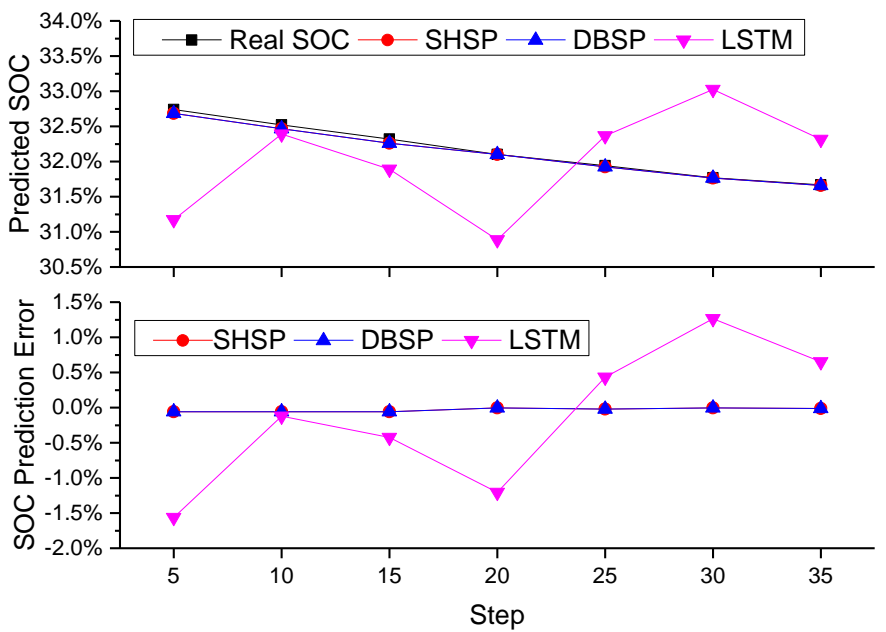

Fig. 6. 7 different steps forward SOC prediction comparisons in the trip with $32.9 \%$ initial SOC

The maximum absolute prediction error of SHSP and DBSP is around $0.06 \%$. The levels of prediction accuracy of both methods are similar. The maximum prediction error of LSTM is more than $1.5 \%$. The prediction error of SHSP is 25 times less than that of LSTM. SHSP achieves higher accuracy than LSTM-based SOC prediction, since LSTM is trained by the historical SOC information and is easily affected by trained data.

\section{E. Influence of Temperature on Prediction Performance}

The influence of temperature on the time of running the three methods is studied. Fig. 7 shows the time of running the three methods is similar at four different temperatures. SHSP is the most time-saving method compared with DBSP and LSTM at four different temperatures. Hence, the temperature has a limited effect on the time of running SHSP.

With respect to the SOC prediction accuracy, Fig. 8 shows SOC prediction results under temperatures with 35 prediction steps. All the curves can follow the trend of the real SOC. The curves of SHSP and DBSP are closer than LSTM to the curve of the real SOC.

In terms of the prediction error, with estimated power, the prediction errors of SHSP under 4 different temperatures are similar, which are around $0.55 \%$. This prediction accuracy is close to that of DBSP. If the errors of the estimated power are removed in this discharge, assuming precise power in the future discharge is known in advance, the SOC prediction errors are 
about $0.03 \%$ by using SHSP. This result is also close to the accuracy of DBSP. The prediction accuracy of SHSP with estimated power approximately increases $45 \%$ compared with LSTM. Generally, the prediction accuracy of SHSP is stable and acceptable at different temperatures.

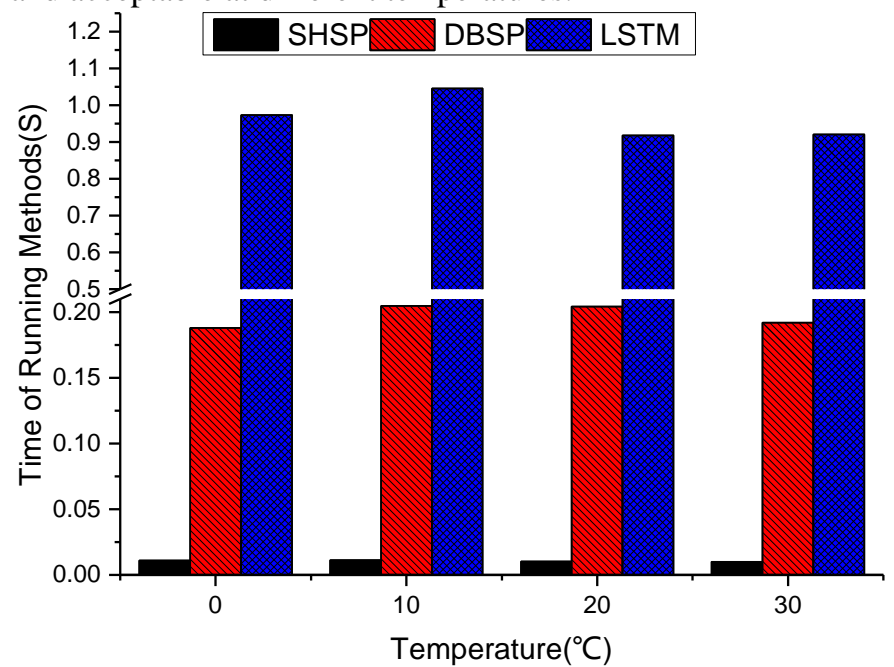

Fig. 7. Time of running the three methods at different temperature

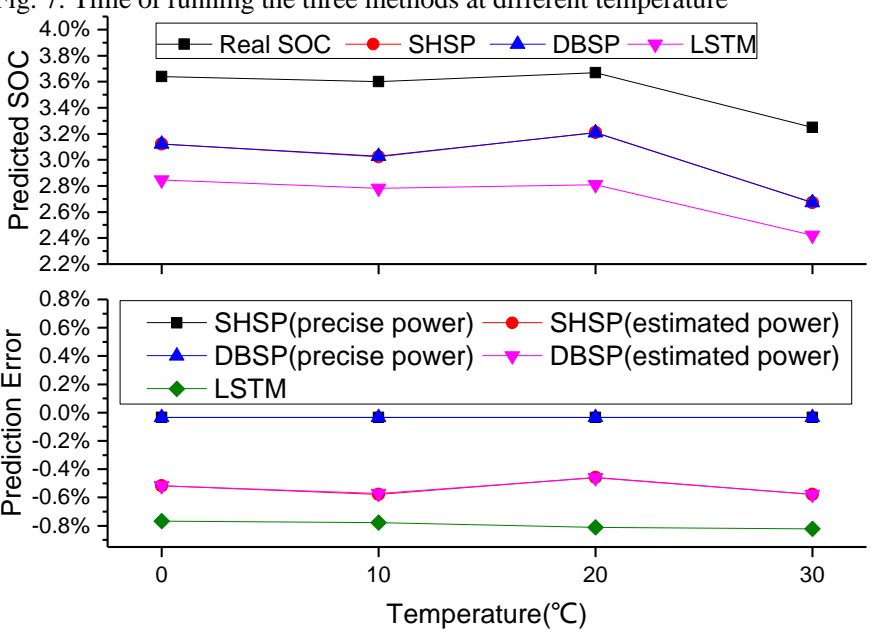

Fig. 8. SOC prediction at different temperatures at 35 prediction steps

\section{CONCLUSIONS}

We have developed a simplified SOC prediction algorithm based on driving information to alleviate range anxiety. This paper established two Rint-based models with a constant capacitor of a supercapacitor. Based on the two models, the analytical expression relating the historical average power and predicted SOC is proposed. Finally, the SHSP is compared with DBSP and LSTM via experiments. The experimental results show that the 35-step forward prediction error of SHSP is close to that of DBSP and much lower than that of LSTM. This means that the predictor can help drivers to know what the SOC will be at the destination and whether they can approach the end. Importantly, SHSP saves around 94\% time of DBSP, 98\% time of LSTM.

Because of low computing cost, SHSP is possibly applied in a mobile application to help EV drivers to decide charge nearby or keep driving. It could even be adopted for route planning of EVs in automatic pilot, especially when the remaining capacity of energy storage elements is low. In future work, we will enrich the study with the consideration of stochastic consideration, for example, traffic influence.

\section{APPENDIX}

\section{CURRENT IN THE EQUIVALENT CIRCUIT OF A SUPERCAPACITOR UNDER CONSTANT DISCHARGE}

The derivation of formulas (16) and (17) will be calculated based on the circuit in Fig. 2, and the calculation is referenced from [27].

According to the Rint-based model of supercapacitors, formula (25) is achieved. Then formula (26) is obtained according to the Kirchhoff's Voltage Law.

$$
\begin{gathered}
I_{P}(t)=-C \frac{d U_{c}(t)}{d t}=\frac{P_{\mathrm{ha}}}{U_{P}(t)} \\
U_{c}(t)=r_{P} I_{P}(t)+U_{P}(t)
\end{gathered}
$$

Rearranging (25) and (26) and then acquiring a first-order, second-degree differential equation (27) in consideration of discharge power $P_{\text {ha }}>0$.

$$
\dot{U}_{c}^{2}+\frac{1}{r_{P} C} U_{c} \dot{U}_{c}+\frac{P_{\mathrm{ha}}}{r_{P} C^{2}}=0
$$

In order to solve this equation, equation (27) is rewritten by using its two roots $\lambda_{1}, \lambda_{2}$, as equation (28), provided the internal potential $U_{c}(\mathrm{t})>2 \sqrt{r_{P} P_{\text {ha }}}$. The roots $\lambda_{1}, \lambda_{2}$ are expanded in equation (29).

$$
\begin{gathered}
\dot{U}_{c}^{2}+\frac{1}{r_{P} C} U_{c} \dot{U}_{c}+\frac{P_{\mathrm{ha}}}{r_{P} C^{2}}=\left(\dot{U}_{c}+\lambda_{1}\right)\left(\dot{U}_{c}+\lambda_{2}\right) \\
\dot{U}_{c}^{2}+\frac{1}{r_{P} C} U_{c} \dot{U}_{c}+\frac{P_{\mathrm{ha}}}{r_{P} C^{2}} \\
=\left(\dot{U}_{c}+\frac{U_{c}}{2 r_{P} C}-\sqrt{\left.\left(\frac{U_{c}}{2 r_{P} C}\right)^{2}-\frac{P_{\mathrm{ha}}}{r_{P} C^{2}}\right)\left(\dot{U}_{c}+\frac{U_{c}}{2 r_{P} C}+\sqrt{\left(\frac{U_{c}}{2 r_{P} C}\right)^{2}-\frac{P_{\mathrm{ha}}}{r_{P} C^{2}}}\right)}\right.
\end{gathered}
$$

Let $a=2 \sqrt{r_{P} P_{\mathrm{ha}}}$ and $u=U_{c}$ such that the radical for the first root in (29) can be simplified as follows:

$$
\frac{d t}{2 r_{P} C}=-\frac{u}{a^{2}} d u-\frac{\sqrt{u^{2}-a^{2}}}{a^{2}} d u
$$

Equation (30) can be integrated as shown in (31).

$$
\int_{0^{+}}^{t} \frac{d \tau}{2 r_{P} C}=-\int_{U_{c 0}}^{U_{c}} \frac{u}{a^{2}} d u-\int_{U_{c 0}}^{U_{c}} \frac{\sqrt{u^{2}-a^{2}}}{a^{2}} d u
$$

The full solution to the constant power discharge of the supercapacitor is obtained in (32) by making a further change to the variables and simplifying the expression.

$$
\begin{aligned}
t= & \frac{C}{4 P_{\mathrm{ha}}}\left[U_{c 0}^{2}-U_{c}^{2}\right]+r_{P} C \ln \left\{\frac{U_{c 0} \sqrt{U_{c 0}^{2}-4 r_{P} P_{\mathrm{ha}}}}{U_{c} \sqrt{U_{c}^{2}-4 r_{P} P_{\mathrm{ha}}}}\right. \\
& +\frac{C}{4 P_{\mathrm{ha}}}\left[U_{c 0} \sqrt{U_{c 0}^{2}-4 r_{P} P_{\mathrm{ha}}}-U_{c} \sqrt{U_{c}^{2}-4 r_{P} P_{\mathrm{ha}}}\right]
\end{aligned}
$$

The discharge current $I_{P}(t)$ under constant power loading is finally solved, shown in (33).

$$
I_{P}(t)=-C \dot{U}_{c}(t)=\frac{U_{c}(t)}{2 r_{P}}-\sqrt{\left(\frac{U_{c}(t)}{2 r_{P}}\right)^{2}-\frac{P_{\mathrm{ha}}}{r_{P}}}
$$




\section{REFERENCES}

[1] R. Lu, C. Zhu, L. Tian, and Q. Wang, "Super-Capacitor Stacks Management System With Dynamic Equalization Techniques," in international symposium on electromagnetic launch technology, 2007, vol. 43, no. 1, pp. 254-258.

[2] A. Ostadi and M. Kazerani, "A Comparative Analysis of Optimal Sizing of Battery-Only, UltracapacitorOnly, and Battery-Ultracapacitor Hybrid Energy Storage Systems for a City Bus," IEEE Transactions on Vehicular Technology, vol. 64, no. 10, pp. 44494460, 2015.

[3] L. Mir, I. Etxeberriaotadui, I. P. De Arenaza, I. Sarasola, and T. Nieva, "A supercapacitor based light rail vehicle: system design and operations modes," in energy conversion congress and exposition, 2009, pp. 1632-1639.

[4] Z. Li, C. Zhu, J. Jiang, K. Song, and G. Wei, "A 3-kW Wireless Power Transfer System for Sightseeing Car Supercapacitor Charge," IEEE Transactions on Power Electronics, vol. 32, no. 5, pp. 1-1, 2016.

[5] F. Naseri, E. Farjah, T. Ghanbari, Z. Kazemi, E. Schaltz, and J. Schanen, "Online Parameter Estimation for Supercapacitor State-of-Energy and State-ofHealth Determination in Vehicular Applications," IEEE Transactions on Industrial Electronics, pp. 1-1, 2019.

[6] P. Saha, S. Dey, and M. Khanra, "Modeling and Stateof-Charge Estimation of Supercapacitor Considering Leakage Effect," IEEE Transactions on Industrial Electronics, pp. 1-1, 2019.

[7] P. Saha, S. Dey, and M. Khanra, "Accurate estimation of state-of-charge of supercapacitor under uncertain leakage and open circuit voltage map," Journal of Power Sources, vol. 434, no. SEP.15, pp. 226696.1226696.8, 2019.

[8] P. Saha, S. Dey, and M. Khanra, "Accurate estimation of state-of-charge of supercapacitor under uncertain leakage and open circuit voltage map," Journal of Power Sources, vol. 434, p. 226696, 2019.

[9] J. Wang, L. Zhang, J. Mao, J. Zhou, and D. Xu, "Fractional order equivalent circuit model and SoC estimation of supercapacitors for use in HESS," IEEE Access, vol. 7, pp. 52565-52572, 2019.

[10] M. Ceraolo, G. Lutzemberger, and D. Poli, "State-OfCharge Evaluation Of Supercapacitors," Journal of Energy Storage, vol. 11, pp. 211-218, Jun 2017.

[11] B. Homan, M. V. T. Kortenaar, J. L. Hurink, and G. J. M. Smit, "A realistic model for battery state of charge prediction in energy management simulation tools," Energy, vol. 171, pp. 205-217, 2019.

[12] I. Yaqoob, L. U. Khan, S. A. Kazmi, M. Imran, N. Guizani, and C. S. Hong, "Autonomous driving cars in smart cities: Recent advances, requirements, and challenges," IEEE Network, vol. 34, no. 1, pp. 174-181, 2019.

[13] S. Kaffash, A. T. Nguyen, and J. Zhu, "Big data algorithms and applications in intelligent transportation system: A review and bibliometric analysis," International Journal of Production Economics, vol. 231, p. 107868, 2021.

[14] A. Neilson, B. Daniel, and S. Tjandra, "Systematic review of the literature on big data in the transportation domain: Concepts and applications," Big Data Research, vol. 17, pp. 35-44, 2019.

[15] Y.-W. Huang, P.-C. Lin, and J. Wang, "The influence of bus and taxi drivers' public self-consciousness and social anxiety on aberrant driving behaviors," Accident Analysis \& Prevention, vol. 117, pp. 145-153, 2018.

[16] A. Dineva, B. Csomós, S. K. Sz, and I. Vajda, "Investigation of the performance of direct forecasting strategy using machine learning in State-of-Charge prediction of Li-ion batteries exposed to dynamic loads," Journal of Energy Storage, vol. 36, p. 102351, 2021.

[17] J. Hong, Z. Wang, W. Chen, L. Y. Wang, and C. Qu, "Online joint-prediction of multi-forward-step battery SOC using LSTM neural networks and multiple linear regression for real-world electric vehicles," The Journal of Energy Storage, vol. 30, p. 101459, 2020.

[18] H. Wang, G. Zhou, R. Xue, Y. Lu, and J. A. McCann, "A Driving-Behavior-Based SoC Prediction Method for Light Urban Vehicles Powered by Supercapacitors," IEEE Transactions on Intelligent Transportation Systems, pp. 1-10, 2019.

[19] Y. Wang, C. Liu, R. Pan, and Z. Chen, "Modeling and state-of-charge prediction of lithium-ion battery and ultracapacitor hybrids with a co-estimator," Energy, vol. 121, no. FEB.15, pp. 739-750, 2017.

[20] Z. Song, J. Ho, H. E. Hofmann, X. Lin, and J. Sun, "Parameter Identification and Maximum Power Estimation of Battery/Supercapacitor Hybrid Energy Storage System Based on Cramer-Rao Bound Analysis," IEEE Transactions on Power Electronics, vol. 34, no. 5, pp. 4831-4843, 2019.

[21] J. Zhang, Z. Wang, P. Liu, and Z. Zhang, "Energy consumption analysis and prediction of electric vehicles based on real-world driving data," Applied Energy, vol. 275, p. 115408, 2020.

[22] C. Zhang, X. Yu, G. Dong, J. Wei, and Z. Chen, "A method for remaining discharge time prediction of lithium-ion batteries under dynamic uncertainty," International Journal of Energy Research, vol. 43, no. 5, pp. 1760-1774, 2019.

[23] C. Wang, H. He, Y. Zhang, and H. Mu, "A comparative study on the applicability of ultracapacitor models for electric vehicles under different temperatures," Applied Energy, vol. 196, pp. 268-278, 2017.

[24] H. Yang and Y. Zhang, "Characterization of supercapacitor models for analyzing supercapacitors connected to constant power elements," Journal of Power Sources, vol. 312, pp. 165-171, 2016.

[25] G. E. Forsythe, "Computer methods for mathematical computations," Prentice-Hall series in automatic computation, vol. 259, 1977.

[26] L. Zubieta and R. Bonert, "Characterization of double- 
layer capacitors for power electronics applications," IEEE Transactions on Industry Applications, vol. 36, no. 1, pp. 199-205, 1997.

[27] J. M. Miller, Ultracapacitor Applications. Institution of Engineering and Technology, 2011.

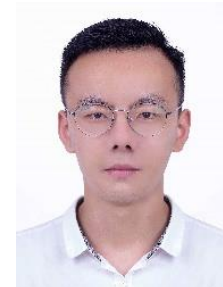

Houlian Wang received the Ph.D. degree in mechatronic engineering from China University of Mining and Technology, Xuzhou, China, in 2019.

He was also a Visiting Ph.D. Student with Imperial College London, London, U.K. He is currently working with the School of Mechanical Engineering, Jiangsu University of Science and Technology, Zhenjiang, China. His research interests include battery management.

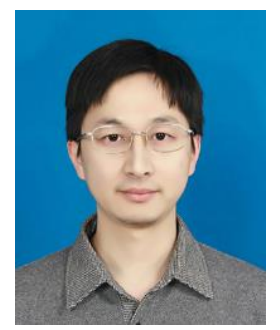

Gongbo Zhou received the B.S. degree in computer science and technology from the Anhui University of Science and Technology, in 2005, and the D.E. degree from the School of Mechanical and Electrical Engineering, China University of Mining and Technology, Xuzhou, China, in 2010.

From 2009 to 2010, he was a visiting Ph.D. student with University of Wisconsin, Madison, WI, USA. He is currently a Professor with the School of Mechatronic Engineering, China University of Mining and Technology. His current research interests include battery management and wireless sensor networks.

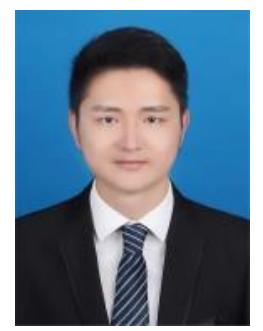

Jing $\mathbf{X u}$ is an associate professor of mechatronic engineering at Marine Equipment and Technology Institute, Jiangsu University of Science and Technology, Zhenjiang, China.

He received the Ph.D. degree from China University of Mining and Technology, Xuzhou, China, in 2018. His research interests include signal processing, pattern recognition and intelligent system.

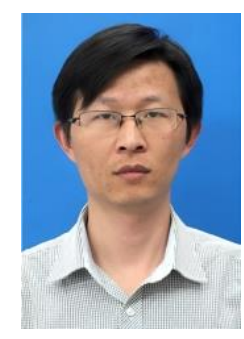

Zhiqiang Liu received the Ph.D. degree in mechanical engineering from Shanghai Jiao Tong University, Shanghai, China, in 2018. $\mathrm{He}$ is currently working with the School of Mechanical Engineering, Jiangsu University of Science and Technology, Zhenjiang, China. His research interests include battery management and energy storage.

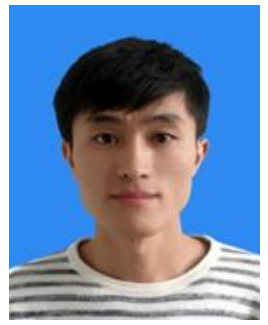

Xiaodong Yan received a B.S. degree from Yancheng Institute of Technology in 2018. At present, he is currently pursuing the Ph.D. degree in mechanical engineering from China University of Mining and Technology. His research interests include piezoelectric vibration energy harvesting technology, wireless sensor networks and battery state of charge prediction.

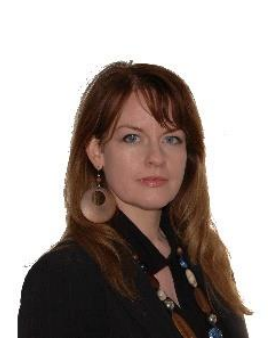

Julie A McCann is a Full-Professor of Computing Systems at Imperial College London, Department of Computing. Her research centres on highly decentralized and self-organizing scalable algorithms for sensor-based computing systems, wireless networks and energy neutral computing.

She leads the Adaptive Embedded Systems Engineering Research Group and has worked with Intel, Cisco and NEC etc. on substantive smart city projects. She is an elected peer for the EPSRC and is a Fellow of the BCS. 